\title{
Análisis de los efectos en la capacidad funcional de adultos mayores de 65-75 años de edad mediante el sistema de realidad virtual IREX
}

\section{Analysis of the effects on the functional capacity of adults over 65-75 years old through the IREX virtual reality system}

\author{
ALMARAZ-MOCTEZUMA, Sofía del Carmen†**, SÁNCHEZ-BARRERA, Erendira y VÁZQUEZ- \\ CHACÓN, Verónica \\ ID $1^{\text {er }}$ Autor: Sofía del Carmen, Almaraz-Moctezuma \\ ID $1^{\mathrm{er}}$ Coautor: Erendira, Sánchez-Barrera / ORC ID: 0000-0003-2466-6977, arXiv Author ID: Erendiris_28 \\ ID $2^{\text {do }}$ Coautor: Verónica, Vázquez-Chacón / ORC ID: 0000-0003-2002-6876, Researcher ID Thomson: V-5652-2018,
} arXiv Author ID: Veronica_\#77

DOI: $10.35429 /$ JP.2019.10.3.14.20

Recibido 18 de Octubre, 2019; Aceptado 13 de Diciembre, 2019

\section{Resumen}

Objetivo: Analizar los efectos del sistema de realidad virtual IREX en la capacidad funcional de adultos mayores de 65-75 años de edad. Metodología: Se aplicó un estudio clínico con 15 adultos mayores. Se solicitó a los participantes su consentimiento libre e informado para participar en el estudio. Se aplicaron 18 sesiones en total por un período de 6 semanas, 3 sesiones a la semana. Cada sesión fue basada en incrementar la capacidad funcional mediante la incorporación de realidad virtual. Se realizó un tratamiento combinado de ejercicios con el soporte del sistema de realidad virtual IREX. Contribución: Según los resultados, el sistema de realidad virtual IREX facilita la inserción social del paciente, otorgándole una mayor autonomía para la realización de sus actividades de la vida diaria, mejorando su calidad de vida y aumentando su capacidad funcional, dando a los terapeutas una visión de los aspectos más relevantes sobre el sistema de realidad virtual IREX y su aplicación en el campo de la rehabilitación..

Adulto mayor, Realidad virtual, Capacidad funcional

\begin{abstract}
Objective: To analyze the effects of the IREX virtual reality system on the functional capacity of adults over 65-75 years old. Methodology: A clinical study with 15 older adults was applied. Participants were asked for their free and informed consent to participate in the study. A total of 18 sessions were applied for a period of 6 weeks, 3 sessions per week. Each session was based on increasing functional capacity by incorporating virtual reality. A combined exercise treatment was carried out with the support of the IREX virtual reality system. Contribution: According to the results, the IREX virtual reality system facilitates the social insertion of the patient, granting greater autonomy to carry out their daily life activities, improving their quality of life and increasing their functional capacity, giving therapists a vision of the most relevant aspects of the IREX virtual reality system and its application in the field of rehabilitation.
\end{abstract}

Senior, Virtual reality, Functional capacity

Citación: ALMARAZ-MOCTEZUMA, Sofía del Carmen, SÁNCHEZ-BARRERA, Erendira y VÁZQUEZ-CHACÓN, Verónica. Análisis de los efectos en la capacidad funcional de adultos mayores de 65-75 años de edad mediante el sistema de realidad virtual IREX. Revista de Fisioterapia. 2019. 3-10: 14-20

\footnotetext{
* Correspondence to Author (email: soff_a_m@live.com.mx)

$\uparrow$ Researcher contributing first author.
} 


\section{Introducción}

En todo el mundo, se ha incrementado el número de personas que llegan a la tercera edad. México presenta un envejecimiento progresivo con un incremento marcado en los últimos años; por cada diez mexicanos, uno es un adulto mayor ( $10 \%$ de la población total). Se estima que en el año 2030 la población mexicana de 60 años y más aumente a 20 millones ( $14.8 \%$ de la población nacional) y en 2050 represente al $21.5 \%$ de los mexicanos (1).

La capacidad funcional del adulto mayor es definida como el conjunto de habilidades físicas, mentales y sociales que permiten al sujeto la realización de las actividades que exige su medio y/o entorno, si la capacidad funcional se ve alterada se produce pérdida de independencia (2). En los adultos mayores de manera natural existen múltiples cambios morfológicos y fisiológicos que pueden alterar la capacidad funcional. En el aspecto físico, la OMS refiere que las personas mayores de 65 años de edad son quienes sufren un mayor número de caídas mortales (3).

Las caídas en ancianos son un importante problema de salud pública ya que son la principal causa de morbilidad, pérdida de independencia y muerte en individuos mayores de 65 años, lo que genera altos costos de medicamentos, rehabilitación, hospitalización e institucionalización (4).

Se aplicó el sistema IREX que significa Immersive Rehabilitation Exercise por sus siglas en inglés, que permite colocar a pacientes en ambientes virtuales de deporte o de juego, donde se guían a través de regímenes de ejercicio terapéutico. El terapeuta es el encargado de diseñar un programa de ejercicios divertido e interactivo que involucra movimientos de determinados segmentos o la función de todo el cuerpo.

La implementación de este tipo de terapia puede disminuir en gran medida el umbral de discapacidad y así reducir el nivel de deterioro de los adultos mayores debido a que mediante un sistema de realidad virtual se puede simular una escena real en la que ofrece retroalimentación visual y sensorial, a través de la simulación de ejercicios que el paciente tendrá que hacer para la rehabilitación física y cognitiva.

\section{Metodología}

Se realizó un estudio de tipo longitudinal con un diseño cuasiexperimental en los meses Octubre - Enero 2019 en el Centro Médico de Alta Especialidad Gb Rehabilitación.

La muestra quedó constituida por 15 pacientes que según su historia clínica, no presentan enfermedades cognitivas, neurodegenerativas, ni alteraciones visuales o auditivas.

Para realizar el estudio se utilizó el sistema de realidad virtual IREX que consta de una cámara conectada a una computadora y de un guante rojo inalámbrico, conectados a un televisor para ver la imagen más grande en una habitación verde.

Al iniciar el tratamiento se realizó una entrevista para conocer la capacidad funcional del paciente mediante la evaluación de las Actividades Básicas de la Vida Diaria (ABVD) con el Índice de Barthel y la evaluación de Actividades Instrumentales de la Vida Diaria (AIVD) con la escala de Lawton y Brody. También se evaluó el estado funcional del equilibrio de los pacientes mediante la Berg Balance Scale y la calidad de vida con la escala GENCAT. Al finalizar el tratamiento se volvió a realizar este proceso.

El tratamiento consta de 18 sesiones; 3 por semana (lunes, miércoles y viernes) en un período de 6 semanas. Cada sesión fue basada en favorecer la capacidad funcional $y$ el equilibrio con la aplicación del sistema de realidad virtual. En cada sesión se utilizaron 6 programas terapéuticos diferentes, con duración de 5 minutos cada programa y descansos de 2 minutos entre estos, estableciendo entonces las sesiones con un tiempo definido de 40 minutos.

Se dividió el tratamiento en dos fases; En la primera fase, los lunes se realizaban ejercicios de miembro superior y equilibrio, los miércoles ejercicios de miembro inferior y los viernes se usaban aplicaciones de equilibrio con miembro superior. En la segunda fase, es decir, de la sesión 10 - 18 se comenzaron a utilizar dos aplicaciones de cada área con mayor dificultad. 


\section{Instrumentos a recolectar y métodos de control}

$\begin{array}{ll}\text { - } & \text { Sistema IREX (consta de cámara } \\ \text { conectada a una computadora y de un } \\ \text { guante) }\end{array}$

\section{Análisis y resultados}

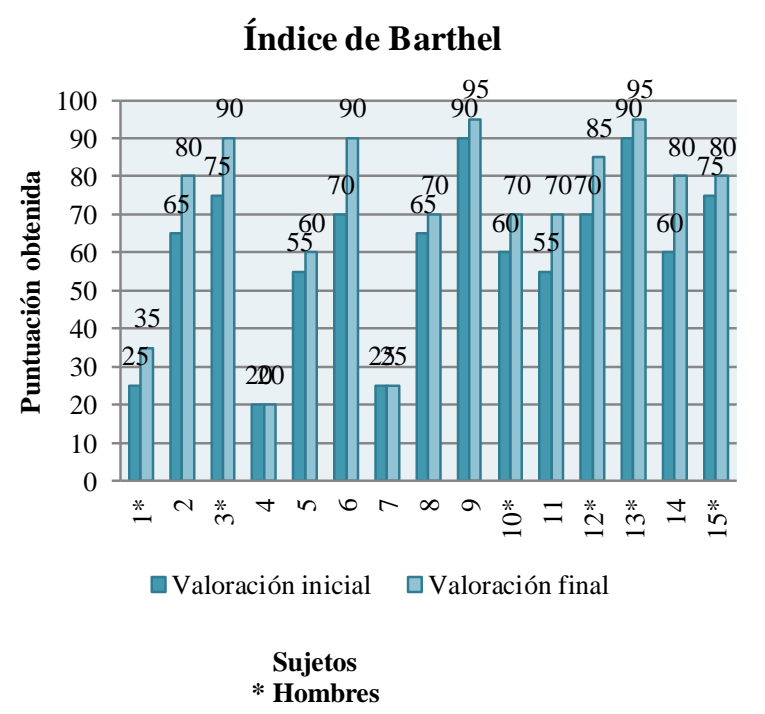

Gráfico 1 Evaluación de las ABVD mediante el Índice de Barthel, valores obtenidos al inicio y al final del tratamiento

Trece de los quince sujetos $(86.6 \%)$ aumentaron al menos 5 puntos, mientras los dos adultos mayores restantes (13.3\%), presentaron los valores más bajos en la valoración inicial y mantuvieron esos valores al final. El sexo y la edad no parecen afectar los resultados de esta encuesta.

Tres sujetos $(20 \%)$ se mantuvieron en dependencia grave, dos $(13.3 \%)$ pasaron de dependencia moderada a leve, ocho $(53.3 \%)$ se mantuvieron en dependencia leve, y dos (13.3\%) pasaron de dependencia leve a independencia.

\section{Escala de Lawton y Brody}

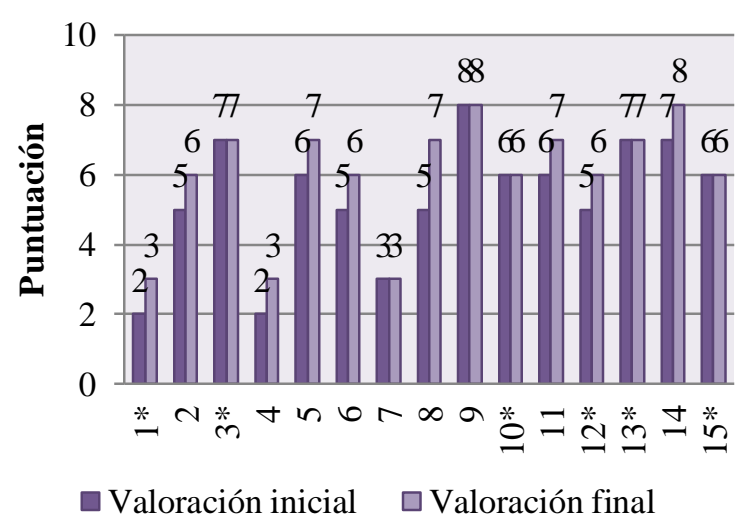

Sujetos

*Hombres

Gráfico 2 Evaluación de las AIVD mediante la Escala de Lawton y Brody, valores obtenidos al inicio y al final del tratamiento

Nueve de los quince sujetos $(60 \%)$ aumentaron al menos 1 punto, los seis adultos mayores restantes $(40 \% \%)$, presentaron los mismos valores en la valoración inicial y final. De esos seis pacientes, 4 son hombres $(66.6 \%)$ por lo que se infiere que en realidad esos pacientes no realizan algunas de las actividades que se evalúan porque no quieren, y no porque no pueden. Tres sujetos (20\%) se mantuvieron en dependencia severa, cuatro $(26.6 \%)$ pasaron de dependencia moderada a dependencia ligera, seis $(40 \%)$ se mantuvieron en dependencia ligera, uno $(6.6 \%)$ paso de dependencia ligera a independencia, uno $(6.6 \%)$ se mantuvo independiente desde el inicio hasta el final.

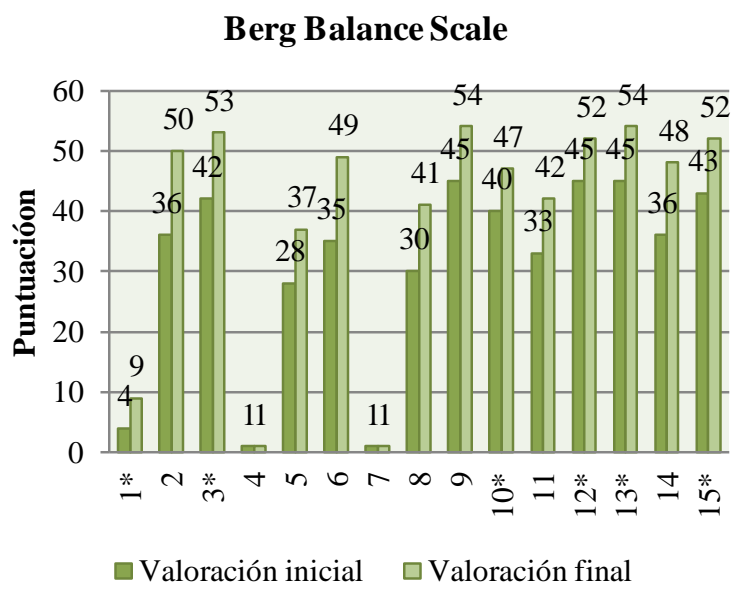

Sujetos

*Hombres

Gráfico 3 Evaluación de la capacidad funcional del equilibrio mediante la Berg Balance Scale, valores obtenidos al inicio y al final del tratamiento 
Trece de los quince sujetos (86.6\%) aumentaron al menos 5 puntos, mientras los dos adultos mayores restantes (13.3\%), presentaron los valores más bajos en la valoración inicial y mantuvieron esos valores al final, debido a que son pacientes que usan silla de ruedas, por lo que no hubo un cambio.

El sexo y la edad no parecen afectar los resultados de esta encuesta. Tres sujetos (20\%) se mantuvieron en un alto riesgo de caída, uno (6.6\%) en moderado riesgo, seis sujetos (40\%) pasaron de moderado riesgo a leve riesgo de caída, y cinco $(33.3 \%)$ se mantuvieron en leve riesgo de caída.

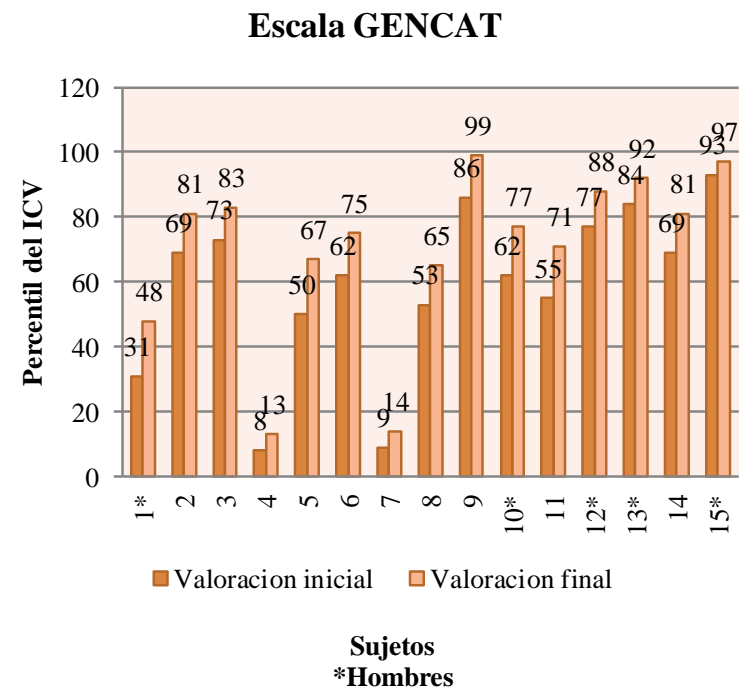

Gráfico 4 Evaluación del Índice de Calidad de vida mediante la escala GENCAT

Todos los sujetos del estudio (100\%) aumentaron su calidad de vida en un porcentaje considerable, por lo que el objetivo se cumplió. Incluso los pacientes que acudieron en silla de ruedas, a diferencia de las otras escalas. El sexo y la edad no parecen afectar los resultados de esta encuesta. Doce de los quince sujetos (80\%) se encuentran en los percentiles altos.

\section{Conclusiones}

La rehabilitación con el uso del sistema de realidad virtual IREX supone un método terapéutico con efectos positivo para aumentar la capacidad funcional en adultos mayores de 65-75 años.
Los programas favorecen la adherencia al tratamiento ya que genera un ambiente lúdico, y menos estresante para el paciente, además, de que el aprendizaje se vuelve intuitivo y autodidacta, se pierde el temor a equivocarse o a saber si están haciendo bien un ejercicio al generar una retroalimentación positiva y en tiempo real al paciente, ofrecen un entorno seguro para realizar la rehabilitación y lo único negativo sería el costo.

En nuestra opinión se requieren estudios con mayor tamaño muestral y con más escalas de medición y características de los pacientes para aportar mayor validez a la intervención en la práctica clínica diaria.

Según los resultados, se concluye que el sistema de realidad virtual IREX facilita la inserción social del paciente, otorgándole una mayor autonomía para la realización de actividades de la vida diaria, mejorando su calidad de vida y aumentando su capacidad funcional.

\section{Referencias}

Avila C, Velarde E. Evaluacion de la calidad de vida. [Articulo de revisión] Salud Publica Mex 2002; [Consultado el 27 de Octubre del 2018] 44:349- 361. Disponible en: http://www.scielo.org.mx/pdf/spm/v44n4/1402 3.pdf

Barragán L. ESCALA BERG: valoración del equilibrio en pacientes con Daño Cerebral Adquirido [Internet]. España: Neuro RHB; [Actualizado el 21 de Septiembre del 2017; Consultado el 23 de Octubre del 2018]. Disponible en: https://neurorhb.com/blog-danocerebral/escala-berg-valoracion-delequilibrioen-pacientes-con-dca/

Barrero CL Solís, García S, Arrioja, Ojeda A. Índice de Barthel (IB): Un instrumento esencial para la evaluación funcional y la rehabilitación. Plast \& Rest Neurol [Revista electrónica]. 2005 [Consultado el 20 de Octubre del 2018]; 4 (2): 81-85. Disponible en: http://www.sld.cu/galerias/pdf/sitios/rehabilitac ion-doc/indice_de_barthel.pdf

Baztán JJ, Pérez J, Alarcón T, San Cristóbal E, Izquierdo G, Manzabeitia I. Índice de Barthel: Instrumento válido para la valoración funcional de pacientes con enfermedad cerebrovascular. 
Revista Española Geriátrica Gerontológica [Revista electrónica]. 1993 [Consultado el 20 de Octubre del 2018]: 28: 32-40.

Berg K, Maki B, Williams Jl, Holliday P, Wood S. A comparison of clinical and laboratory measures of postural balance in an elderly population. Arch Phys Med Rehab [Revista electrónica]. 1992 [Consultado el 23 de Octubre del 2018]; 73 (1) 73-83. 98

Dauphinee S, Berg K, Bravo G, Williams JI. The Balance Scale: Responding to clinically meaningful changes. Canadian Journal of Rehabilitation [Internet]. Canada; 1997 [Consultado el 23 de Octubre del 2018]; 10: 35 50. Disponible

en:

http://www.chiropractic.on.ca/wp-

content/uploads/fp-bergbalance-scale.pdf

Gesture Tek Health. IREX [Internet] USA: 2016 [Consultado el 25 de Octubre del 2018]. Disponible

en:

http://www.gesturetekhealth.com/products/irex

39-Gesture Tek Health. IREX:

BACKGROUND \& HISTORY [Internet] USA:

2016 [Consultado el 25 de Octubre del 2018].

Disponible

en:

http://www.gesturetekhealth.com/sites/default/f

iles/general/irex_concept_not e_0.pdf 40-

Gesture Tek Health. IREX History [Internet] USA: 2016 [Consultado el 25 de Octubre del 2018]. Disponible en: http://www.gesturetekhealth.com/node/55

González KD. Envejecimiento demográfico en México: análisis comparativo entre las entidades federativas [Internet]. México: CONAPO (Consejo Nacional de Población); [Actualizado 04 de Febrero del 2016; Consultado 04 de Octubre del 2018]. Disponible en: http://www.conapo.gob.mx/es/CONAPO/Envejecim iento_demografico_en_Mexico

INEGI (Instituto Nacional de Estadística y Geografía). Esperanza de vida al nacimiento/ Sexo y entidad federativa, 2010 a 2016 [Internet]. México; Agosto 2016 [Consultado en 15 de Octubre del 2018]. Disponible en: http://cuentame.inegi.org.mx/poblacion/esperan za.aspx?tema $=\mathrm{P}$

INEGI. Perfil sociodemográfico de adultos mayores en 2014 [Internet]. México; Noviembre 2014 [Consultado el 15 de Octubre del 2018].
Disponible

en:

http://internet.contenidos.inegi.org.mx/contenid os/productos/prod_serv/contenidos/espanol/bvi negi/productos/censos/poblacion/2010/perfil_so cio/adulto s/702825056643.pdf

Landinez NS, Contreras K, Castro A. Proceso de envejecimiento, ejercicio y fisioterapia. Revista Cubana de Salud Pública [Revista electrónica]. Cuba: 2012 [Consultado el 17 de Octubre del 2018]; 38 (4): 564-565. Disponible en:https://www.scielosp.org/article/ssm/content /raw/?resource_ssm_path=/

media/assets/rcsp/v38n4/spu08412.pdf

Lazcano BG, Rodriguez GR. Evaluación geriátrica multidimensional. Práctica de la Geriatría. 2.a ed. México: Mc-Graw-Hill, 2007, pp. 83-104.

Letona M. La eficacia de la realidad virtual en la rehabilitación de pacientes con ictus [Internet]. España: 2015 [Consultado el 24 de Octubre del 2018]. Disponible en: https://www.unav.edu/documents/29044/62641 19/mp9t3.pdf 100

Leyva BM. Movilidad, equilibrio y caídas en los adultos mayores. Geroinfo [Revista electrónica]. Cuba: 2008 [Consultado el 14 de Octubre del 2018]; 2 (3): 2-3. Disponible en: http://www.sld.cu/galerias/pdf/sitios/gericuba/movil idad,_equilibrio_y_caidas_bibliografia.pdf 95

Lloréns R, Colomer C, Alcañiz M, Noé E. BioTrak virtual reality system: effectiveness and satisfaction analysis for balance rehabilitation in patients with brain injury. Neurologia [Internet]. Junio 2013 [Consultado 24 de Octubre del 2018]; 28 (5): 268-275.

Lobo A, Santos MP, Carvalho J. Anciano institucionalizado: calidad de vida y funcionalidad. Revista Española Geriátrica Gerontológica [Revista electrónica]. 2007; 42 (1): 22-6.

Mahoney FI, Barthel D. Functional evaluation: The Barthel Index. Maryland State Medical Journal [Revista electrónica]. 1965 [Consultado el 20 de Octubre del 2018]; 14: 56-61.97

Medina B, Rodríguez GR, García LA. Abatimiento funcional y falla para recuperarse en función de la funcionalidad. Práctica de la Geriatría. 2.a ed. México: Mc-Graw-Hill, 2007, pp. 130-134.

ALMARAZ-MOCTEZUMA, Sofía del Carmen, SÁNCHEZ-BARRERA, Erendira y VÁZQUEZ-CHACÓN, Verónica. Análisis de los efectos en la capacidad funcional de adultos mayores de 65-75 años de edad mediante el sistema de realidad virtual IREX. Revista de Fisioterapia. 2019 
Navarrete JM. La realidad virtual como arma terapéutica en rehabilitación. Rehabil. Integral [Revista electrónica]. 2010 [Consultado el 24 de Octubre del 2018]; 5 (1): 40-45. Disponible en: https://www.rehabilitacionintegral.cl/wpcontent/files_mf/5navarrete.pdf

Olazarán J, Mouronte P, Bermejo F. Validez clínica de dos escalas de actividades instrumentales en la enfermedad de Alzheimer. Neurología [Revista electrónica]. 2005 [Consultado el 21 de Octubre del 2018]; 20: 395- 401 .

OMS. Hombres, envejecimiento y salud [Internet]. Ginebra; 2001 [Consultado el 19 de Octubre del 2018]. [Consultado el 17 de Octubre del 2018]. Disponible en: http://envejecimiento.csic.es/documentos/docu mentos/oms-hombres-01.rtf

Organización Mundial de la Salud (OMS). Caídas [Internet]; 16 de enero de 2018 [Consultado el 04 de Octubre del 2018]. Disponible en https://www.who.int/es/newsroom/fact-sheets/detail/falls

Organización Panamericana de la Salud. Evaluación funcional del adulto mayor [Internet]. Oficina Regional de la OMS; 2016 [Consultado el 19 de Octubre del 2018]. Disponible en: http://www.sld.cu/galerias/pdf/sitios/gericuba/ modulo3.pdf

PENSIONISSSTE. Día del adulto mayor [Internet]. México; 25 de agosto de 2017 [Consultado el 15 de Octubre del 2018]. Disponible en: https://www.gob.mx/pensionissste/articulos/dia -del-adulto-mayor123010?idiom=es

Peñasco B, Reyes A, Gil A, Bernal A, Pérez- B, Peña A. Aplicación de la realidad virtual en los aspectos motores de la neurorrehabilitación [Revista electrónica]. 2010 [Consultado el 23 de Octubre del 2018]; 51 (8): 281-288.

Reuben DV, Hazzard WR, Blass JP, Halter JB, Ouslander JG, Tinneti ME. Principles of geriatric assessment. 5a ed. USA: Mc Graw Hill; 2007, pp. 99- 118.
Rikli R y cols. Senior Fitness Tets Manual [Internet]. España: Human Kinetic.; 2007 [Consultado el 19 de Octubre del 2018] Disponible en: http://scielo.isciii.es/scielo.php?script=sci_artte xt\&pid=S1134928X2011000400003

Schalock R. L. y Verdugo M. A. El concepto de calidad de vida en los servicios y apoyos para personas con discapacidad intelectual. Siglo Cero [Revista electrónica]. 2007 [Consultado 27 de Octubre del 2018]; 38(4), 21- 36.

Schalock R. L. y Verdugo M. A. Quality of life conceptual and measurement frameworks: from concept to application in the field of intellectual disabilities. 99 Evaluation of Program Planning [Revista electrónica]. 2008 [Consultado 27 de Octubre del 2018]; 31 (2), 181-190.

Segovia MG, Torres EA. Funcionalidad del adulto mayor $\mathrm{y}$ el cuidado enfermero. GEROKOMOS [Revista electrónica]. San Luis Potosí: 2011 [Consultado 04 de Octubre del 2018]; 22 (4): 162-166. Disponible en: http://scielo.isciii.es/pdf/geroko/v22n4/comunic acion2.pdf

Spirduso MR, García NJ. Introducción a la problemática del envejecimiento. Psicomotricidad y ancianidad. García y Morales. Madrid: 96 Ciencias de la educación preescolar y especial; 2004. [Consultado el 17 de Octubre del 2018] pp. 19-27.

STCONAPRA (Secretariado Técnico Consejo Nacional para la Prevención de Accidentes). Modelo para la Prevención de Lesiones por Caídas en Personas Adultas Mayores en México [Internet]. México, Distrito Federal: Secretaría de Salud; 2016. [Consultado el 07 de Octubre del 2018]. Disponible en: https://www.gob.mx/cms/uploads/attachment/fi le/207103/ModeloCaidas2.pdf

Trigás M, Ferreira L, Meijide H. Escalas de valoración funcional en el anciano. Galicia Clin [Revista electrónica]. 2011 [Consultado el 20 de Octubre del 2018]; 72 (1): 11-16. Disponible en: https://galiciaclinica.info/pdf/11/225.pdf

Trigás M. Valoración de la discapacidad física: el índice de Barthel. Revista Española de Salud Pública [Revista electrónica]. Feb 2007 [Consultado el 21 de Octubre del 2018]; 21 (3): $31-34$

Disponible 
https://meiga.info/escalas/ESCALADELAWT ONYBRODY.pdf

Valderrama E, Pérez J. Una visión crítica de las escalas de valoración funcional traducidas al castellano. Rev Esp Geriatria Gerontologia [Revista electrónica]. 1997 [Consultado 23 de Octubre del 2018]; 32 (5): 297-306.

Verdugo M. A., Gómez L. E. y Arias B. Evaluación de la calidad de vida en adultos mayores. 1a ed. Salamanca: Instituto Universitario de Integración en la Comunidad; 2009 [Consultado 27 de Octubre del 2018].

Verdugo M. A., Schalock R. L., Gómez L. E. y Arias B. Construcción de escalas de calidad de vida multidimensionales centradas en el contexto: la Escala GENCAT. Siglo Cero [Revista electrónica]. 2007 [Consultado 27 de Octubre del 2018]; 38(4), 57-72.

Verdugo M.A, Arias B, Gomes L.E, Schalock R.L. Manual de la aplicación de la escala GENCAT de calidad de vida. [Internet]. Barcelona; Enero 2009 [Consultado el 27 de Octubre del 2018]. Disponible en: http://inico.usal.es/documentos/EscalaGencatM anualCAST.pdf 